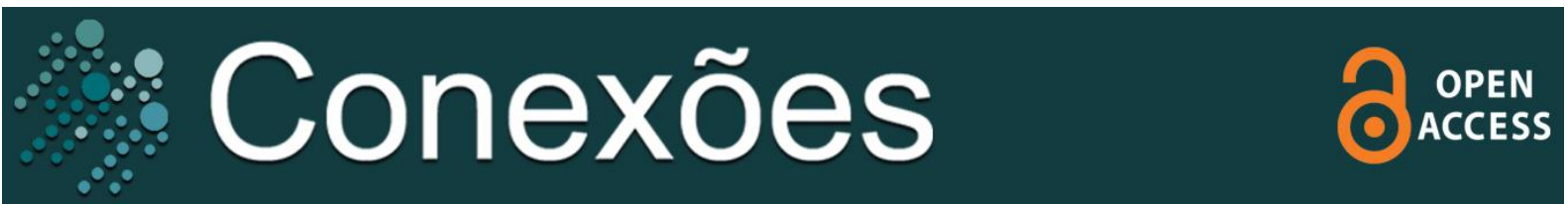

Artigo Original

\title{
Motivos que levam praticantes e não praticantes de exercício físico do Distrito Federal a não se exercitarem em academias
}

\author{
Reasons that lead practitioners and non-practitioners of physical exercise in \\ the Federal District (Brazil) not to exercise in gyms
}
Razones que llevan a practicantes y no practicantes de ejercicio físico del Distrito Federal (Brazil) a no ejercitarse en gimnasios

\author{
Henrique de Oliveira Castro ${ }^{1}$ \\ Dirce Rodrigues Caixeta ${ }^{2}$ \\ Luan Martins de Mesquita Gonçalves ${ }^{2}$ \\ Luiz Eduardo Alves Rodrigues ${ }^{2}$ \\ Sérgio Avelino da Silva ${ }^{2}$ \\ Vivian de Oliveira²
}

\begin{abstract}
RESUMO
Objetivo: Identificar os motivos que levam praticantes e não praticantes de exercícios físicos do Distrito Federal (DF) a não procurarem uma academia para se exercitarem. Metodologia: Participaram 223 moradores do DF, de ambos os sexos, com idades entre 18 e 66 anos, que não frequentam academias. Os voluntários foram divididos em quatro grupos de acordo com o sexo e prática de atividades físicas. Foi utilizado um questionário estruturado, elaborado pelos próprios pesquisadores, que contém 16 perguntas. Resultados e discussão: De forma geral, os homens que não praticam exercícios físicos em academias não o fazem por falta de tempo $(40,7 \%)$ e por indisposição $(36,4 \%)$, seguido por questões financeiras, não gostar do ambiente de academia e outros motivos. Já as mulheres, os principais motivos foram não gostarem do ambiente da academia $(45,7 \%)$ e indisposição $(41,9 \%)$, seguido por falta de tempo, questões financeiras e outros motivos. Conclusão: Conclui-se que, para homens praticantes de exercício, os principais motivos para não procurarem uma academia para se exercitar são as questões financeiras e a falta de tempo. Já para as mulheres praticantes de exercício, os principais motivos são não gostar do ambiente de academia e a indisposição.
\end{abstract}

Palavras-chave: Exercício físico. Academias de Ginástica. Motivação.

\footnotetext{
1 Universidade Federal de Mato Grosso, Faculdade de Educação Física, Departamento de Educação Física, Cuiabá - MT, Brasil.

2 Centro Universitário Instituto de Educação Superior de Brasília, Departamento de Educação Física, Brasília - DF, Brasil.
}

\section{Correspondência:}

Henrique de Oliveira Castro. Universidade Federal de Mato Grosso, Departamento de Educação Física, Av. Fernando Corrêa da Costa, 2367, Boa Esperança, Cuiabá - MT, CEP 78060-900. Email: henriquecastro88@yahoo.com.br 


\begin{abstract}
Objective: Identify the reasons that lead practitioners and non-practitioners of physical exercises in the Federal District (DF, Brazil) not to look for a gym to exercise. Methodology: Participated 223 DF residents of both sexes, aged between 18 and 66 years, who do not attend gyms. Volunteers were divided in four groups, according to sex and physical activity practice. A structured questionnaire, prepared by the researches, was used, contains 16 questions. Results and discussion: In general, men who do not practice physical exercise in gyms do not do it due to lack of time $(40.7 \%)$ and indisposition $(36.4 \%)$, followed by financial questions, not to like the gym environment and other reasons. As for women, the main reasons were not to like the gym environment $(45.7 \%)$ and indisposition ( $41.9 \%$ ) followed by lack of time, financial questions and other reasons. Conclusion: It is concluded that for men who exercise, the main reasons for not looking for a gym to exercise are financial questions and lack of time. For women who exercise, the main reasons are not to like the gym environment and the indisposition.
\end{abstract}

Keywords: Physical Exercise. Fitness Centers. Motivation.

\title{
RESUMEN
}

Objetivo: Identificar las razones que llevan a practicantes y no practicantes de ejercicios físicos del Distrito Federal (DF, Brazil) a no buscar un gimnasio para hacer ejercicio. Metodología: Participaron 223 residentes del DF, de ambos sexos, con edades comprendidas entre 18 y 66 años, que no asisten a gimnasios. Los voluntarios fueron divididos en cuatro grupos según el sexo y practica de la actividad física. Se utilizó un cuestionario estructurado, elaborado por los proprios investigadores, que contenía 16 preguntas. Resultados y discusión: En general, los hombres que no practican ejercicio físico en gimnasios no lo hacen por falta de tiempo $(40,7 \%)$ y indisposición $(36,4 \%)$, seguidos de cuestiones económicas, no les gustar el ambiente del gimnasio y otras razones. En cuanto a las mujeres, las principales razones fueron que no les gusta el ambiente del gimnasio $(45,7 \%)$ y la indisposición $(41,9 \%)$, seguidas de la falta de tiempo, problemas económicos y otras razones. Conclusión: Se concluye que, para los hombres que hacen ejercicio, las principales razones para no buscar un gimnasio para ejercitarse son las cuesiones económicas y la falta de tiempo. Para las mujeres que hacen ejercicio, las principales razones son que no les gusta el ambiente del gymnasio y la indisposición.

Palabras Clave: Ejercício Físico. Centros de Acondicionamiento. Motivación. 


\section{INTRODUÇÃO}

A literatura tem apresentado que um indivíduo regularmente ativo melhora a qualidade e a duração da sua vida (BURNS; MURRAY, 2014; MARTIN et al., 2009). A prática regular de exercícios físicos possui um importante papel na prevenção e na diminuição do risco de mortalidade, cardiopatias, hipertensão, diabetes tipo II, osteoporose, obesidade, câncer de cólon e mama (EMMCOLLISON et al., 2020; KYU et al., 2016; MARTIN et al., 2009; REBAR et al., 2015; WHO, 2020). O exercício físico também reduz danos psicológicos como a ansiedade, depressão, declínios cognitivos e níveis de estresse, além de melhorar o bem-estar geral e mental (BERNARD et al., 2015; LEE et al., 2015). Desta forma, programas de exercícios físicos são sugeridos como necessários para promover estilos de vida saudáveis e uma melhor qualidade de vida (HAWKES et al., 2014).

As recomendações atuais da Organização Mundial da Saúde (WHO, 2020) para adultos (18 a 64 anos de idade), indicam que todos os adultos devem praticar atividade física regular, sendo pelo menos 150 a 300 minutos de atividade aeróbica de intensidade moderada ou pelo menos 75 a 150 minutos de atividade aeróbica de intensidade vigorosa ou uma combinação equivalente de atividade física de intensidade moderada e vigorosa ao longo da semana para benefícios substanciais à saúde. Além disso, adultos devem realizar também atividades de fortalecimento muscular de intensidade moderada ou maior, que envolvam os principais grupos musculares dois ou mais dias por semana para benefícios adicionais à saúde (WHO, 2020). Contudo, evidências indicam que $43 \%$ dos adultos não atendem às recomendações mínimas de exercícios físicos (HALLAL et al., 2012; KAPTEYN et al., 2018).

De acordo com a Organização Mundial da Saúde (2014), o sedentarismo ocupa o quarto lugar como um dos principais fatores de risco para a mortalidade, sendo um fator de preocupação para os sistemas de saúde. Para Bueno e colaboradores (2016), os elevados índices de inatividade física da população tornase onerosa à economia, independente do país ou região considerada, já que está relacionada com o alto gasto com medicamentos e custos hospitalares e clínicos, como internações e consultas. Dados do IBGE (2015) indicam que $62,1 \%$ da população brasileira é sedentária. No Distrito Federal, a quantidade de pessoas sedentárias representa $49,6 \%$ da população.

Os principais motivos que levam os sujeitos de diferentes faixas etárias a não praticarem exercícios físicos regularmente estão atrelados, principalmente, à falta de tempo, longa jornada de trabalho ou estudos, falta de motivação ou vontade, dores, atendimento profissional desqualificado, poucos aparelhos para a prática, cansaço, ocorrência de lesões, não promoção da socialização entre os participantes, monotonia, distância do local de prática, necessidade de horário fixo para prática, demora na percepção de resultados e doenças na família 
(DASKAPAN; TUZUN; EKER, 2006; DUNTON; SCHNEIDER, 2006; LIZ; ANDRADE, 2016; REICHERT et al., 2007).

Apesar dos desafios, a busca por programas de exercícios físicos vem aumentando em diversas faixas etárias e em diferentes populações, sendo as academias de ginástica uma opção para a realização desses programas (BARTHOLOMEU NETO et al., 2013). Considerando que a vida cotidiana e suas comodidades induzem a diminuição do movimento e o aumento do sedentarismo, as academias são alternativas para manter uma vida ativa, por meio de exercícios físicos programados e supervisionados, buscando a melhoria do desempenho ou aptidão física, estética, saúde, bem-estar físico, mental, interação social e lazer (LEITÃo et al., 2000).

Apesar do expressivo aumento de academias de ginástica no Brasil a partir da década de 80, a taxa de permanência de alunos nos programas de exercício físico em academias é baixa (BARTHOLOMEU NETO et al., 2013). Destaca-se ainda, um grande número de pessoas sedentárias e, segundo Weinberg e Gould (2017), o problema enfrentado pelos profissionais de saúde e de exercícios físicos é conseguir que estas pessoas comecem a praticar exercícios, fazendo desta, uma prática constante no cotidiano das pessoas (LIZ; ANDRADE, 2016).

Balbinotti e Capozzoli (2008) apontam que um dos principais motivos para esse crescimento de praticantes de exercícios em academias é a busca por saúde. Por outro lado, observa-se que grande parte dos trabalhos publicados sobre a temática relacionam-se aos motivos do abandono da prática de exercícios físicos.

Lopes e colaboradores (2016) indicam que a identificação de barreiras para o exercício físico é altamente pertinente para as intervenções, uma vez que o reconhecimento destas pode guiar os programas e suas ações, de forma a definir metodologias e estratégias mais eficientes. Por esse motivo, o presente estudo foca em identificar os motivos que levam praticantes e não praticantes de exercícios físicos do Distrito Federal a não procurarem uma academia como opção para se exercitarem. Ao conhecer os motivos que justificam o afastamento destes grupos de indivíduos das academias, pode-se compreender melhor suas necessidades, assim como planejar estratégias específicas para o atendimento orientado a esse nicho do mercado do exercício físico e saúde.

\section{MÉTODO}

\section{AMOSTRA}

Trata-se de uma pesquisa qualitativa (THOMAS; NELSON; SILVERMAN, 2012), de caráter descritivo, que buscou compreender os motivos que levam as pessoas a não praticar exercícios físicos em academias. 
A população do presente estudo foi composta por 223 voluntários de ambos os sexos (118 homens e 105 mulheres) com idades entre 18 e 66 anos. Como critério de inclusão no presente estudo, os voluntários teriam que ser adultos (acima de 18 anos de idade), moradores do Distrito Federal (DF), praticantes e não praticantes de exercício físico regular, e que não frequentam academias. A amostra foi selecionada de maneira não probabilística intencional (THOMAS; NELSON; SILVERMAN, 2012), pois a intencionalidade garantiu que todos os grupos preenchessem os critérios necessários à sua formação.

Os voluntários foram divididos em quatro grupos: Grupo Mulheres Praticantes - GMP ( $n=48$, idade média de 41,5 $\pm 13,9$ anos, praticantes de exercício físico regular a pelo menos um ano e não frequentadoras de academias), Grupo Mulheres Não Praticantes - GMNP ( $n=57$, idade média de 41,5 $\pm 14,5$ anos, não praticantes de exercício físico), Grupo Homens Praticantes - GHP ( $n=56$, idade média de 38,3 $\pm 14,7$ anos, praticantes de exercício físico regular a pelo menos um ano e não frequentadores de academias) e Grupo Homens Não Praticantes - GHNP ( $n=62$, idade média de $38,1 \pm 14,1$ anos, não praticantes de exercício físico).

\section{INSTRUMENTO}

Foi utilizado um questionário estruturado, elaborado pelos próprios autores, contendo 16 perguntas ( 11 abertas e 5 fechadas). O questionário foi escrito no Google Forms com o objetivo de facilitar o envio para os voluntários participantes do presente estudo.

As primeiras questões foram relativas às características da amostra (nome, idade, sexo e local onde reside). Para a análise das variáveis do estudo, ou seja, das questões específicas, os voluntários tinham que responder duas questões fechadas: se eram praticantes de exercício físico regular a pelo menos um ano e se frequentam academia para se exercitar (ambas com opções para resposta "sim" ou "não"), e uma questão aberta: por qual motivo não frequenta a academia para se exercitar (nesta, o voluntário tinha total liberdade de escrita em sua resposta).

Os motivos que levam as pessoas a não frequentarem as academias para se exercitarem foram incluídos em cinco categorias, sendo: "falta de tempo" (em geral ou por motivo de trabalho/estudo), "indisposição" (cansaço, sedentarismo, preguiça, falta de coragem, falta de vontade, falta de interesse e falta de incentivo), "questões financeiras" (falta de dinheiro, preços exorbitantes, cobrança a maior para pagamentos mensais), "não gosto do ambiente da academia" (prefiro atividades ao ar livre, não suporto, monotonia das atividades, som muito alto, ambiente hostil, não gosto de lugar fechado, higienização dos aparelhos, ambiente fechado e sem estímulo, muito repetitivo, bate papo dos treinadores com os alunos em assuntos totalmente alheios aos exercícios) e "outros" (prefiro outros tipos de exercícios físicos, gosto mais de esportes coletivos, prefiro realizar os exercícios 
em casa, demora na obtenção de resultados, falta de priorizar atividade física).

\section{Procedimentos}

O presente estudo encontra-se em concordância com as normas estabelecidas pelo Conselho Nacional de Saúde (Res. CNS 196/96) e Declaração de Helsinque de 1975 para pesquisas com seres humanos, e aprovado pelo Comitê de Ética em Pesquisas da Universidade sob CAAE n 86780518.8.0000.5083.

Inicialmente, com o intuito de explicar a pesquisa e convidar para a participação, foi realizado contato prévio com 270 moradores do DF através de telefone (43), e-mail (20), redes sociais - Instagram e Facebook (66) e WhatsApp (141). Esse contato foi realizado por três pesquisadores responsáveis pelo estudo, e foi criado um roteiro para escrita e fala homogêneas durante as abordagens aos possíveis voluntários. Cada pesquisador ficou responsável pelo contato com 90 possíveis voluntários, selecionados dentro do círculo de contatos dos mesmos.

Após o contato, 223 voluntários aceitaram participar do estudo. Dos 47 que não participaram, os motivos foram: por não responderem ou retornarem ao contato, ou por não terem interesse no estudo. Para os 223 voluntários que compuseram a amostra, os pesquisadores enviaram o Termo de Consentimento Livre e Esclarecido para leitura e concordância dos voluntários que aceitaram participar do estudo. Em seguida, foi enviado pelo WhatsApp e e-mail um link do Google Forms contendo o questionário com as perguntas relativas a pesquisa. No momento do preenchimento do questionário, os voluntários respondiam sozinhos, porém, se houvesse qualquer dúvida, poderiam contatar o pesquisador responsável para esclarecimentos. Esse contato era realizado via WhatsApp ou ligação telefônica, ficando à critério do voluntário. O pesquisador ficava disponível para o voluntário durante todo o preenchimento. Todos os procedimentos utilizados no estudo foram padronizados utilizando-se do roteiro criado pelos pesquisadores.

\section{ANÁLISE DOS DADOS}

As respostas do questionário foram tabuladas em uma planilha para a realização de uma análise descritiva dos dados, através de percentis, utilizandose o software Exce/ ${ }^{\circledR} 2003$.

\section{Resultados e Discussão}

A Tabela 1, apresenta os resultados das análises das respostas dos voluntários do sexo masculino em relação aos motivos indicados para não praticarem exercícios físicos em academias. 
Tabela 1 - Motivos indicados pelos grupos de homens para não praticarem exercícios em academias. Valores expressos em quantidade de respostas e percentual

\begin{tabular}{ccccccc}
\hline Grupos & $\mathbf{N}$ & $\begin{array}{c}\text { Falta de } \\
\text { tempo }\end{array}$ & Indisposição & $\begin{array}{c}\text { Não gosto do } \\
\text { ambiente de } \\
\text { academia }\end{array}$ & $\begin{array}{c}\text { Questões } \\
\text { financeiras }\end{array}$ & Outros \\
\hline GHP & 56 & $9(16 \%)$ & $24(42,8 \%)$ & $17(30,3 \%)$ & $24(42,8 \%)$ & 15 \\
GHNP & 62 & $39(62,9 \%)$ & $19(30,6 \%)$ & $16(25,8 \%)$ & $14(22,6 \%)$ & $8(12,9 \%)$ \\
GERAL & 118 & $48(40,7 \%)$ & $43(36,4 \%)$ & $33(28 \%)$ & $38(32,2 \%)$ & 23 \\
$(19,5 \%)$
\end{tabular}

GHP = Grupo Homens Praticantes; GHNP = Grupo Homens Não Praticantes

De acordo com a Tabela 1, observa-se que, de forma geral, os homens que não praticam exercícios físicos em academias não o fazem por falta de tempo $(40,7 \%)$ e por indisposição $(36,4 \%)$, seguido por questões financeiras, não gostar do ambiente de academia e outros motivos. Do sexo masculino, os homens que praticam exercício fora da academia (GHP), não se exercitam em academias por indisposição $(42,8 \%)$ e questões financeiras $(42,8 \%)$, seguido por não gostar do ambiente de academia, outros motivos e, por último, falta de tempo. Já o grupo dos homens que não praticam exercícios (GHNP), os principais motivos para não procurarem uma academia são a falta de tempo $(62,9 \%)$ e indisposição $(30,6 \%)$, seguido por não gostar do ambiente de academia, questões financeiras e outros motivos.

Sobre as questões financeiras, a literatura aponta alguns resultados conflitantes com os alcançados por este estudo. Hallal e colaboradores (2012), em um estudo realizado com adultos e adolescentes de 105 países, puderam afirmar que a inatividade física é maior nos países com renda elevada. Além disso, Alves e colaboradores (2012), que investigaram os índices de sedentarismo na cidade de Salvador (Bahia, Brasil) verificaram que os jovens com níveis econômicos mais baixos se apresentam mais ativos, quando comparados aos adolescentes com melhores condições financeiras.

Por outro lado, Ferraro e Cândido (2017) investigaram por meio de uma revisão de literatura a percepção dos idosos sobre a atividade física, e descobriram que as condições financeiras são apontadas como o principal empecilho para a aderência às atividades físicas.

No que diz respeito ao fator tempo disponível, resultados semelhantes foram encontrados no estudo realizado por Laurenzano e Loch (2012), que demonstraram que a falta de tempo, a indisposição e a falta de dinheiro foram os principais motivos que levaram homens a abandonar os programas de exercício físico. A indisposição, relacionada a fatores como a falta de estímulo e monotonia, 
também foi apontado no estudo realizado por Liz e Andrade (2016) como um dos motivos mais frequentes de desistência da prática de musculação em academias.

Outro motivo decisivo para o abandono de academias de ginástica por universitários com sobrepeso ou obesos, descrito por Bartholomeu Neto e colaboradores (2013), foi a desmotivação, apresentada como a falta de motivação pessoal e a falta de aulas motivantes. Esses resultados não estão de acordo com os do presente estudo, e essa diferença pode ser explicada pelas características das populações estudadas.

Entre os homens não praticantes, pondera-se a alegação de falta de tempo como motivo principal do afastamento das academias. Esse resultado também é relatado no estudo de Bartholomeu Neto e colaboradores (2013) que o associa ao ritmo acelerado em que a sociedade moderna vive. Denota-se, das respostas apresentadas, que este grupo até demonstra a intenção de fazer exercícios físicos e conhecem os seus efeitos benéficos para a saúde, mas não conseguem adequar suas rotinas de modo a ter tempo disponível para tal prática.

A falta de tempo também foi apontada como o principal fator para a desistência da prática de exercício físico no estudo de Santos e Kniknik (2006). Essa falta de tempo muitas vezes está associada à jornada excessiva de trabalho, às obrigações familiares e a dificuldades na organização do tempo da vida pessoal e profissional.

A Tabela 2, apresenta os resultados das análises das respostas das voluntárias do sexo feminino em relação aos motivos que não praticam exercícios físicos em academias.

Tabela 2 - Motivos indicados pelos grupos de mulheres para não praticarem exercícios em academias. Valores expressos em quantidade de respostas e percentual

\begin{tabular}{ccccccc}
\hline Grupos & $\mathbf{N}$ & $\begin{array}{c}\text { Falta de } \\
\text { tempo }\end{array}$ & Indisposição & $\begin{array}{c}\text { Não gosto do } \\
\text { ambiente de } \\
\text { academia }\end{array}$ & $\begin{array}{c}\text { Questões } \\
\text { financeiras }\end{array}$ & Outros \\
\hline GMP & 48 & $9(18,7 \%)$ & $6(12,5 \%)$ & $36(75 \%)$ & $7(14,6 \%)$ & $4(8,3 \%)$ \\
GMNP & 57 & $14(24,5 \%)$ & $38(66,6 \%)$ & $12(21 \%)$ & $16(28 \%)$ & 6 \\
GERAL & 105 & $23(21,9 \%)$ & $44(41,9 \%)$ & $48(45,7 \%)$ & $23(21,9 \%)$ & 10 \\
$(9,5 \%)$
\end{tabular}

GMP = Grupo Mulheres Praticantes; GMNP = Grupo Mulheres Não Praticantes.

Como demonstrado na Tabela 2, de forma geral, as mulheres que não praticam exercícios físicos em academias não o fazem por não gostarem do ambiente da academia $(45,7 \%)$ e indisposição $(41,9 \%)$, seguido por falta de tempo, questões financeiras e outros motivos. Destas, as mulheres que praticam 
exercício fora da academia (GMP), não se exercitam em academias por não gostarem do ambiente (75\%) e falta de tempo $(18,7 \%)$, seguido por questões financeiras, indisposição e outros motivos. Já o grupo das mulheres que não praticam exercícios (GMNP), os principais motivos para não procurarem uma academia são a indisposição $(66,6 \%)$ e questões financeiras $(28 \%)$, seguido por falta de tempo, não gostar do ambiente e outros motivos.

Compreender os motivos apontados pelas mulheres é de vital importância, já que a literatura aponta que os índices de inatividade física em mulheres são maiores quando comparados aos homens, em diferentes faixas etárias (BARUFALDI et al., 2012; BASTOS; ARAUJO; HALLAL, 2008; EMM-COLLISON et al., 2020; HALLAL et al., 2012; KAPTEYN et al., 2018).

Em um estudo realizado por Ornelas e colaboradores (2019), os autores indicaram uma tendência das mulheres de considerar uma quantidade maior de barreiras para a prática de exercício físico quando comparadas aos homens. Essas barreiras são relacionadas à ansiedade física ou social, cansaço, falta de tempo ou instalações, o que diminui a aderência desse grupo ao exercício físico. Assim, os dados apresentados pelos autores sugerem a relação de fatores sociais com a não adesão à atividade física. Em relação a diferença entre os sexos, os papeis sociais exercidos por homens e mulheres influenciam diretamente nos modelos de sociedade em que vivemos (ZANCHETTA et al., 2010).

O fato das mulheres indicarem que não gostam do ambiente pode estar associado à falta de conhecimento das atividades oferecidas pelas academias, fato que, segundo Lopes e colaboradores (2016), pode contribuir para uma acomodação e até mesmo gerar a percepção de incapacidade. Além disso, a percepção do ambiente pode estar associada com estereótipos pré-concebidos.

Laurenzano e Loch (2012) apresentam resultados semelhantes ao avaliarem os motivos que levam mulheres a desistência da prática de programas de exercício físico, sendo o desânimo, o cansaço e a preguiça (classificados em nosso estudo como "indisposição") os principais motivos para tal. Outros motivos mencionados no estudo de Laurenzano e Loch (2012) foram a falta de tempo, a falta de companhia e questões financeiras, o que também está de acordo com os resultados do presente estudo. Também corroboram com este estudo os achados de Walsh e Simpson (2020), que investigaram as barreiras para o exercício percebidas por 355 mulheres irlandesas, com idades entre 30 e 55 anos. As autoras afirmam que, de forma bastante evidente, a barreira mais indicada a pelas pesquisadas foi a falta de tempo.

É importante ressaltar que a indisposição não pode ser considerada um sinônimo de preguiça, pois a motivação para a adesão à prática regular de exercícios físicos pode ser fruto de inúmeros fatores como demográfico, biológico, social, emocional, comportamental e cultural, ou seja, elementos intrínsecos e 
extrínsecos do indivíduo (FONTANA et al., 2013; TAVARES JUNIOR; PLANCHE, 2016).

Algumas limitações do estudo são apontadas, como a não validação do instrumento utilizado e a falta de um maior detalhamento de alguns itens, como "não gosto do ambiente de academia", com o objetivo de especificar o que do ambiente que as pessoas não gostam. Sugere-se, para estudos futuros, a validação do questionário utilizado, e sua aplicação em diversas populações, de diferentes idades, locais e nível socioeconômico, a fim de ser traçado os perfis de motivos de não adesão à prática de exercícios físicos em academias.

\section{CONSIDERAÇÕES FINAIS}

Conclui-se que, para homens que praticam exercícios, os principais motivos para não procurarem uma academia para se exercitar são as questões financeiras e a falta de tempo. Já para as mulheres que praticam exercícios, os principais motivos são não gostar do ambiente de academia e a indisposição.

Os resultados deste estudo auxiliam na percepção de clientes em relação ao ambiente de academias para a prática de exercícios físicos. Assim, repensar a questão do ambiente da academia e como torná-la mais atrativa para os clientes e possíveis clientes (principalmente do sexo feminino), pode ensejar todo um campo de novos negócios para os profissionais da área.

\section{NOTAS}

\section{CONFLITOS DE INTERESSE}

Os autores não têm conflitos de interesse, incluindo interesses financeiros específicos e relacionamentos e afiliações relevantes ao tema ou materiais discutidos no manuscrito.

\section{Autoria e CoAutoria}

A autoria é responsável pelos conteúdos do texto. Os autores declaram que participaram de forma significativa na construção e formação desde estudo, tendo, enquanto autor, responsabilidade pública pelo conteúdo deste, pois, contribuíram diretamente para o conteúdo intelectual deste trabalho e satisfazem as exigências de autoria.

Henrique de Oliveira Castro - Concepção e desenvolvimento (desde a ideia para a investigação ou artigo, criou a hipótese); Desenho metodológico (planejamento dos métodos para gerar os resultados); Supervisão (responsável pela organização e execução do projeto e da escrita do manuscrito); Coleta e tratamento dos dados (responsável pelos experimentos, pacientes, organização dos dados); Análise / interpretação (responsável 
pela análise estatística, avaliação e apresentação dos resultados); Levantamento da literatura (participou da pesquisa bibliográfica e levantamento de artigos); Redação (responsável por escrever uma parte substantiva do manuscrito); Revisão crítica (responsável pela revisão do conteúdo intelectual do manuscrito antes da apresentação final).

Dirce Rodrigues Caixeta - Desenho metodológico (planejamento dos métodos para gerar os resultados); Supervisão (responsável pela organização e execução do projeto e da escrita do manuscrito); Coleta e tratamento dos dados (responsável pelos experimentos, pacientes, organização dos dados); Levantamento da literatura (participou da pesquisa bibliográfica e levantamento de artigos); Redação (responsável por escrever uma parte substantiva do manuscrito).

Luan Martins de Mesquita Gonçalves - Desenho metodológico (planejamento dos métodos para gerar os resultados); Supervisão (responsável pela organização e execução do projeto e da escrita do manuscrito); Coleta e tratamento dos dados (responsável pelos experimentos, pacientes, organização dos dados); Levantamento da literatura (participou da pesquisa bibliográfica e levantamento de artigos); Redação (responsável por escrever uma parte substantiva do manuscrito).

Luiz Eduardo Alves Rodrigues - Desenho metodológico (planejamento dos métodos para gerar os resultados); Supervisão (responsável pela organização e execução do projeto e da escrita do manuscrito); Coleta e tratamento dos dados (responsável pelos experimentos, pacientes, organização dos dados); Levantamento da literatura (participou da pesquisa bibliográfica e levantamento de artigos); Redação (responsável por escrever uma parte substantiva do manuscrito).

Sérgio Avelino da Silva - Supervisão (responsável pela organização e execução do projeto e da escrita do manuscrito); Coleta e tratamento dos dados (responsável pelos experimentos, pacientes, organização dos dados); Análise / interpretação (responsável pela análise estatística, avaliação e apresentação dos resultados); Redação (responsável por escrever uma parte substantiva do manuscrito); Revisão crítica (responsável pela revisão do conteúdo intelectual do manuscrito antes da apresentação final).

Vivian de Oliveira - Desenho metodológico (planejamento dos métodos para gerar os resultados); Supervisão (responsável pela organização e execução do projeto e da escrita do manuscrito); Coleta e tratamento dos dados (responsável pelos experimentos, pacientes, organização dos dados); Análise / interpretação (responsável pela análise estatística, avaliação e apresentação dos resultados); Levantamento da literatura (participou da pesquisa bibliográfica e levantamento de artigos); Redação (responsável por escrever uma parte substantiva do manuscrito); Revisão crítica (responsável pela revisão do conteúdo intelectual do manuscrito antes da apresentação final).

\section{REFERÊNCIAS}

ALVES, Carlos Fernando de Amorim; SILVA, Rita de Cássia Ribeiro; ASSIS, Ana Marlúcia Oliveira; SOUZA, Carine de Oliveira; PINTO, Elizabete de Jesus; FRAINER, Deivis Elton Schlickmann. Fatores associados à inatividade física em adolescentes de 10-14 anos de idade, matriculados na rede pública de ensino do município de Salvador, BA. Revista 
Brasileira de Epidemiologia, v. 15, n. 4, p. 858-870, 2012. Disponível em: https://www.scielo.br/pdf/rbepid/v15n4/en 16.pdf. Acesso em: 12 fev. 2020.

BALBINOTTI, Marcos Alencar Abaide; CAPOZZOLI, Carla Josefa. Motivação à prática regular de atividade física: um estudo exploratório com praticantes em academias de ginástica. Revista Brasileira de Educação Física e Esporte, v. 22, n. 1, p. 63-80, 2008. Disponível em: https://www.scielo.br/pdf/epsic/v16n1/a13v16n1. Acesso em: 12 fev. 2020.

BARTHOLOMEU NETO, João; PINTO, Juliane Sousa; SILVA, Junior Vagner Pereira; SALES, Marcelo Magalhães; CÓRDOVA, Cláudio; PEREIRA, Lilian Alves; ASANO, Ricardo YukiO. Motivos do abandono de academias de ginástica por universitários com sobrepeso ou obesidade. Revista Brasileira de Ciência e Movimento, v. 21, n. 3, p. 96-104, 2013. Disponível em: https://portalrevistas.ucb.br/index.php/RBCM/article/view/3714/2756. Acesso em: 12 fev. 2020.

BARUFALDI, Laura Augusta; ABREU, Gabriela de Azevedo; COUTINHO, Evandro Silva Freire; BLOCH, Katia Vergetti. Meta-analysis of the prevalence of physical inactivity among brazilian adolescents. Caderno de Saúde Pública, v. 28, n. 6, p. 1019-1032, 2012. Disponível em: https://www.scielo.br/pdf/csp/v28n6/02.pdf. Acesso em: 12 fev. 2020.

BASTOS, Juliano Peixoto; ARAUJO, Cora Luiza Pavin; HALLAL, Pedro Curi. Prevalence of insufficient physical activity and associated factors in Brazilian adolescents. Journal of Physical Activity and Health, v. 5, n. 6, p. 777-794, 2008. Disponível em: https://pubmed.ncbi.nlm.nih.gov/19164815/. Acesso em: 12 fev. 2020.

BERNARD, Paquito; NINOT, Grégory; BERNARD, Pierre-Louis; PICOT, Marie-Christine; JAUSSENT, Audrey; TAULO, Guillaume; BLAIN, Hubert. Effects of a six-month walking intervention on depression in inactive post-menopausal women: a randomized controlled trial. Aging \& Mental Health, v. 19, n. 6, p. 485-492, 2015. Disponível em: https://www.tandfonline.com/doi/abs/10.1080/13607863.2014.948806. Acesso em: 12 fev. 2020.

BUENO, Denise Rodrigues; MARUCCI, Maria de Fátima Nunes; CODOGNO, Jamile Sanches; ROEDIGER, Manuela de Almeida. Os custos da inatividade física no mundo: estudo de revisão. Ciência \& Saúde Coletiva, v. 21, n. 4, p. 1001-1010, 2016. Disponível em: https://www.scielo.br/pdf/csc/v21n4/1413-8123-csc-21-04-1001.pdf. Acesso em: 12 fev. 2020.

BURNS, Sir Harry.; MURRAY, Andrew Duncan. Creating health through physical activity. British Journal of Sports Medicine, v. 48, n. 3, p. 167-169, 2014. Disponível em: https://bjsm.bmj.com/content/48/3/167. Acesso em: 12 fev. 2020.

DASKAPAN, Arzu; TUZUN, Emine Handan; EKER, Levent. Perceived barriers to physical activity in university students. Journal of Sports Science and Medicine, v. 5, p. 615-620, 2006. Disponível em: https://www.ncbi.nlm.nih.gov/pmc/articles/PMC3861763/. Acesso em: 12 fev. 2020.

DUNTON, Genevieve F.; SCHNEIDER, Margaret. Perceived barriers to walking for physical activity. Preventing Chronic Disease, v. 3, n. 4, p. 1-11, 2006. Disponível em: https://www.ncbi.nlm.nih.gov/pmc/articles/PMC1779280/. Acesso em: 12 fev. 2020.

EMM-COLLISON, Ligia G.; SEBIRE, Simon J.; SALWAY, Ruth; THOMPSON, Janice L; JAGO, Russel. Multidimensional motivation for exercise: A latent profile and transition analysis. Psychology of Sport \& Exercise, v. 47, e101619, 2020. Disponível em: https://www.sciencedirect.com/science/article/pii/S1469029219303851. Acesso em: 12 fev. 2020. 
FERRARO, Naiara Santiago; CÂNDIDO, Aldrina da Silva Confessor. Percepção dos Idosos Acerca da Atividade Física na Terceira Idade. Revista Multidisciplinar e de Psicologia, v. 11, n. 38, 2017. Disponível em: https://idonline.emnuvens.com.br/id/article/view/973. Acesso em: 12 fev. 2020.

FONTANA, Patrícia Silveira; BARBOSA, Marcus Levi Lopes; BALBINOTTI, Marcos Alencar Abaide; BALBINOTTI, Carlos Adelar Abaide. Estudo das motivações à prática da ginástica rítmica: contribuições da pesquisa para o rendimento desportivo. Pensar a Prática, Goiânia, v. 16, n. 2, p. 387-400, 2013. Disponível em:

https://www.revistas.ufg.br/fef/article/view/16873. Acesso em: 12 fev. 2020.

HALLAL, Pedro C.; ANDERSEN, Lars Bo.; BULL, Fiona C.; GUTHOLD, Regina; HASKELL, William; EKELUND, Ulf. Global physical activity levels: surveillance progress, pitfalls, and prospects. The Lancet, v. 380, n. 9838, p. 247-257, 2012. Disponível em:

https://www.thelancet.com/journals/lancet/article/PIIS0140-6736(12)60646-1/fulltext. Acesso em: 12 fev. 2020.

HAWKES, Anna L. PAKENHAM, Kenneth I.; CHAMBERS, Suzanne K.; PATRAO, Tania A.; COURNEYA, Kerry S. Effects of a multiple health behavior change intervention for colorectal cancer survivors on psychosocial outcomes and quality of life: a randomized controlled trial. Annals of Behaviour Medicine, v. 48, n. 3, p. 359-370, 2014. Disponível em: https://pubmed.ncbi.nlm.nih.gov/24722960/. Acesso em: 12 fev. 2020.

IBGE - INSTITUTO BRASILEIRO DE GEOGRAFIA E ESTATÍSTICA. Pesquisa Nacional por Amostra de Domicílios (PNAD). Rio de Janeiro, 2015.

KAPTEYN, Arie; BANKS, James; HAMER, Mark; SMITH, James P. ; STEPTOE, Andrew; VAN SOEST, Arthur; KOSTER, Annemarie; WAH, Saw Htay. What they say and what they do: Comparing physical activity across the USA, England and The Netherlands. Journal of Epidemiology \& Community Health, v. 72, n. 6, p. 471-476, 2018. Disponível em: https://jech.bmj.com/content/jech/early/2018/04/09/jech-2017-209703.full.pdf. Acesso em: 12 fev. 2020.

KYU, Hmwe H.; BACHMAN, Victoria F.; ALEXANDER, Lily T.; MUMFORD, John Everett; AFSHIN, Ashkan; ESTEP, Kara; VERRMAN, John Lennert; DELWICHE, Kristen; LANNARONE, Marissa L.; MOYER, Madeleine L.; CERCY, Kelly; VOS, Theo; MURRAY, Christopher J. L.; FOROUZANFAR, Mohammad H. Physical activity and risk of breast cancer, colon cancer, diabetes, ischemic heart disease, and ischemic stroke events: Systematic review and dose-response meta-analysis for the global burden of disease study 2013. BMJ, v. 354, n. 3857, 2016. Disponível em: https://www.bmj.com/content/354/bmj.i3857. Acesso em: 12 fev. 2020.

LAURENZANO, Michele Juliana; LOCH, Mathias Roberto. Motivos referidos para o abandono de programas de exercício físico ofertados por academias privadas de Presidente Prudente, São Paulo. Arquivos de Ciências do Esporte, Cuiabá, v. 1, n. 1, p. 713, 2012. Disponível em:

http://seer.uftm.edu.br/revistaeletronica/index.php/aces/article/viewFile/244/359. Acesso em: 12 fev. 2020.

LEE, Yunhwan; KIM, Jinhee; HAN, Eun Sook; CHAE, Songi; RYU, Mikyung; AHN, Kwang Ho; PARK, Eun Ju. Changes in physical activity and cognitive decline in older adults living in the community. Age, v. 37, n. 2, p. 20, 2015. Disponível em:

https://www.ncbi.nlm.nih.gov/pmc/articles/PMC4338105/. Acesso em: 12 fev. 2020.

LEITÃO, Marcelo Bichels; LAZZOLI, José Kawazoe; OLIVEIRA, Marcos Aurélio Brazão de; NÓBREGA, Antonio Claudio Lucas da; SILVEIRA, Geraldo Gomes da; CARVALHO, Tales 
de; FERNANDE, Eney Oliveira; LEITE, Neiva; AYUB, Alice Volpe; MICHELS, Glaycon; DRUMMOND, Félix Albuquerque; MAGNI, João Ricardo Turra; MACEDO, Clayton; DE ROSE, Eduardo Henrique. Posicionamento Oficial da Sociedade Brasileira de Medicina do Esporte: Atividade Física e Saúde na Mulher. Revista Brasileira de Medicina do Esporte, v. 6, n. 6, p. 215-220, 2000. Disponível em:

https://www.scielo.br/pdf/rbme/v6n6/v6n6a01.pdf. Acesso em: 12 fev. 2020.

LIZ, Carla Maria de; ANDRADE, Alexandro. Análise qualitativa dos motivos de adesão e desistência da musculação em academias. Revista Brasileira de Ciências do Esporte, v. 38, n. 3, p. 267-274, 2016. Disponível em: https://www.scielo.br/pdf/rbce/v38n3/01013289-rbce-38-03-0267.pdf. Acesso em: 12 fev. 2020.

LOPES, Marize Amorim; KRUG, Rodrigo de Rosso; BONETTI, Albertina; MAZO, Giovana Zarpello. Barreiras que influenciaram a não adoção de atividade física por longevas. Revista Brasileira de Ciências do Esporte, v. 38, n. 1, p. 76-83, 2016. Disponível em: https://www.scielo.br/pdf/rbce/v38n1/0101-3289-rbce-38-01-0076.pdf. Acesso em: 12 fev. 2020.

MARTIN, Corby K; CHURCH, Timothy S.; Thompson, Angela M.; EARNEST, Conrad, P. ; BLAIR, Steven N. Exercise dose and quality of life: a randomized controlled trial. Archives of International Medicine, v. 169, n. 3, p. 269-278, 2009. Disponível em: https://pubmed.ncbi.nlm.nih.gov/19204218/. Acesso em: 12 fev. 2020.

ORGANIZAÇÃO MUNDIAL DE SAÚDE (OMS). Atividade física. N. 385. 2014

ORNELAS, José René Blanco; VALENZUELA, Concepción Soto; HERNÁNDEZ, Zuliana Paola Benitez; FERNÁNDEZ, Fernando Mondaca; GARCÍA, Perla Jannet Jurado. Barreras para la práctica de ejercicio físico en universitarios mexicanos comparaciones por género. Retos, n. 36, p. 80-82, 2019. Disponível em:

https://recyt.fecyt.es/index.php/retos/article/view/67820. Acesso em: 12 fev. 2020.

REBAR, Amanda L; STANTON, Robert; GEARD, David; SHORT, Camille; DUNCAN, Mitch J.; VANDELANOTTE, Corneel. A meta-meta-analysis of the effect of physical activity on depression and anxiety in nonclinical adult populations. Health Psychology Review, v. 9, n. 3, p. 366-378, 2015. Disponível em:

https://www.tandfonline.com/doi/abs/10.1080/17437199.2015.1022901. Acesso em: 12 fev. 2020.

REICHERT, Felipe F.; BARROS, Aluísio J. D.; DOMINGUES, Marlos R.; HALLAL, Pedro C. The role of perceived personal barriers to engagement in leisure time physical activity. American Journal of Public Health, v. 97, p. 515-519, 2007. Disponível em: https://www.ncbi.nlm.nih.gov/pmc/articles/PMC1805028/. Acesso em: 12 fev. 2020.

SANTOS, Susan Cotrim; KNIJNIK, Jorge Dorfman. Motivos de adesão à prática de atividade física na vida adulta intermediária. Revista Mackenzie de Educação Física e Esporte, São Paulo, v. 5, n. 1, p. 23-34, 2006. Disponível em:

http://editorarevistas.mackenzie.br/index.php/remef/article/view/1299. Acesso em: 12 fev. 2020.

TAVARES JUNIOR, Antonio Carlos; PLANCHE, Taynara Citelli. Motivos de adesão de mulheres à prática de exercícios físicos em academias. Revista Equilíbrio Corporal Saúde, v. 8, n. 1, p. 28-32, 2016. Disponível em: https://revista.pgsskroton. com/index.php/reces/article/view/4514. Acesso em: 12 fev. 2020.

THOMAS, Jerry R.; NELSON, Jack K.; SILVERMAN, Stephen J. Métodos de pesquisa em atividade física. 6. ed. Porto Alegre: Artmed, 2012. 
WALSH, Annemarie; SIMPSON, Ellen Elizabeth Anne. Health cognitions mediate physical (in)activity and walking in midlife women. Maturitas, v. 131, p. 14-20, 2020. Disponível em: https://www.sciencedirect.com/science/article/pii/S0378512219302051. Acesso em: 12 fev. 2020.

WEINBERG, Robert S.; GOULD, Daniel. Fundamentos da Psicologia do Esporte e do Exercício. 6. ed. Porto Alegre: Editora Artmed, 2017.

WORLD HEALTH ORGANIZATION (WHO). WHO guidelines on physical activity and sedentary behaviour: at a glance. World Health Organization, 2020.

ZANCHETTA, Luane Margarete; BARROS, Marilisa Berti de Azevedo; CÉSAR, Chester Luiz Galvão; CARANDINA, Luana; GOLDBAUM, Moisés; ALVES, Maria Cecília Goi Porto. Inatividade física e fatores associados em adultos, São Paulo, Brasil. Revista Brasileira de Epidemiologia, v. 13, n. 3, p. 387-399, 2010. Disponível em:

https://www.scielo.br/pdf/rbepid/v13n3/en 03.pdf. Acesso em: 12 fev. 2020.

Recebido em: 03 jul. 2020

Aprovado em: 03 maio 2021

Artigo submetido ao sistema de similaridade Turnitin $®$.

A revista Conexões utiliza a Licença Internacional Creative Commons Atribuição 4.0, preservando assim, a integridade dos artigos em ambiente de acesso aberto.

A Revista Conexões é integrante do Portal de Periódicos Eletrônicos da Unicamp e associado/membro das seguintes instituições:
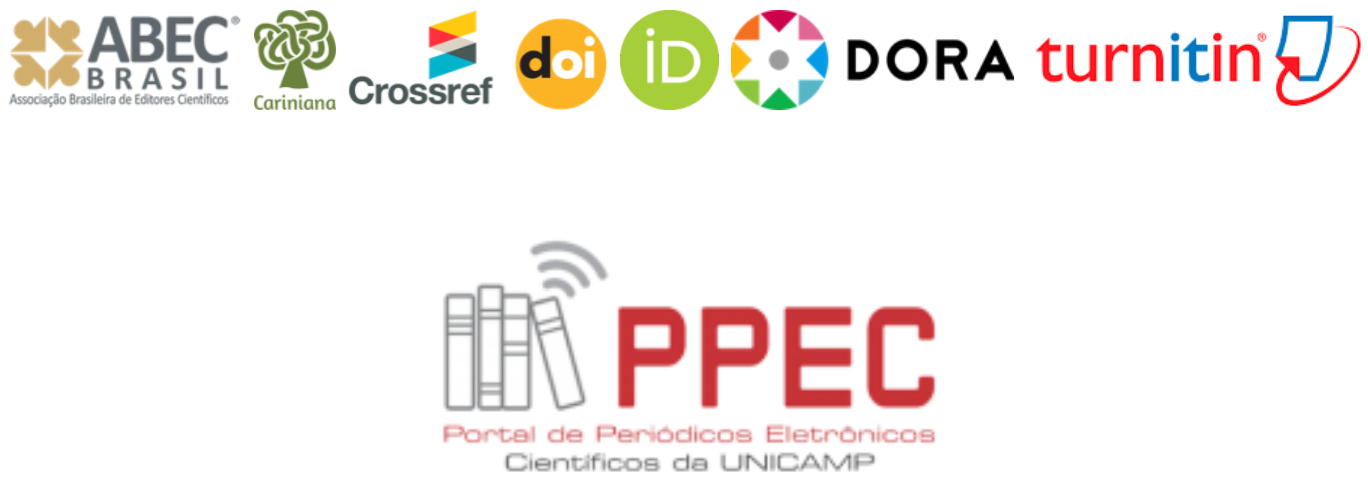